\title{
La preparación psicológica de la Selección Nacional Absoluta de Colombia para el Mundial de Fútbol Brasil 2014
}

\author{
Marcelo Roffé \\ Sociedad Latinoamericana y del Caribe de Psicología de la Actividad Física y del Deporte (SOLCPAD), Argentina
}

RESUMEN:Son pocas las experiencias profesionales del psicólogo del deporteen Selecciones absolutas de fútbolyaún más en un mundial. El objetivo del presente trabajo es el de presentar al lector una experiencia profesional de 3 años con la Selección Absoluta de Colombia, tanto durante el período clasificatorio como en el propio mundial de fútbol de Brasil 2014. La intervención psicológica se ha centrado principalmente en el establecimiento de objetivos, el desarrollo de la cohesión, fortalecimiento de la mente de los jugadores (en las áreas de manejo de las presiones, focalización de la atención y autoconfianza primordialmente) y generar una mentalidad ganadora. Tras 3 mundiales sin participación, Colombia clasifica y queda en 5ta posición. Desde una labor especializada del psicólogo del deporte, se discute la labor realizada y las limitaciones de la intervención, haciendo hincapié en la necesidad de adaptarse al contexto de intervención (concentraciones), la duración de las mismas, la periodicidad entre cada una de ellas y la intervención en el propio mundial, máximo escalón posible al que un futbolista y al que un psicólogo deportivo puede aspirar.

PALABRAS CLAVES: Mundial de fútbol, Intervención Psicológica, Psicólogo del deporte.

\section{The psychological preparation of the Senior Colombian National Team for 2014 World Cup Brazil}

\begin{abstract}
The professional experiences of sport psychologists in Senior National Soccer Teams are limited, and this is even more so the case in a World Cup. The aim of this paper is to present the reader with 3 years of professional experience with the Senior Colombian National Team, both during the qualifying rounds and in the 2014 World Cup Brazil. The psychological intervention focused primarily on establishing objectives, developing cohesion, strengthening the players' minds (in the areas of coping with pressure, attention-focusing and self-confidence, primarily) and generating a winning mentality. After not participating in 3 World Cups, Colombia ranks and remains in 5th place. This sport psychologist's specialized research addresses the work done and the limitations of the intervention, emphasizing the need to adapt to the context of the interventions (concentration), their duration, the time intervals between each of them and intervention during the World Cup itself, the highest level to which a soccer player and a sport psychologist can aspire.
\end{abstract}

KEYWORDS: World Cup, Psychological Intervention, Sport Psychologist.

\section{A preparação psicológica da equipe sênior nacional da Colômbia para a Copa do Mundo Brasil 2014}

RESUMO: Há poucas experiências profissionais do psicólogo do esporte com as seleções sênior no futebol e ainda mais numa Copa do Mundo. O objetivo deste trabalho é apresentar ao leitor uma experiência profissional de 3 anos com a equipe sênior da Colômbia, tanto durante a qualificação como na própria Copa do Mundo Brasil 2014. A intervenção psicológica tem-se focado mormente no estabelecimento de objetivos, o desenvolvimento da coesão, o fortalecimento da mente dos jogadores (primordialmente nas áreas de gerir pressões, focar a atenção e auto-confiança) e gerar uma mentalidade vencedora. Depois de 3 copas do mundo sem obter qualificação, a Colômbia qualifica e atinge a $5^{a}$ posição. Partindo do trabalho especializado do psicólogo do esporte, discute-se esse trabalho realizado e as limitações da intervenção, enfatizando a necessidade de se adaptar ao contexto de intervenção (concentrações), a sua duração, a frequência entre cada uma delas e a intervenção na própria Copa do Mundo, máximo degrau possível ao que um jogador e um psicólogo esportivo podem aspirar.

PALAVRAS-CHAVE: Copa do Mundo, intervenção psicológica, psicólogo do esporte.

Artículo recibido: 20/03/2016 | Artículo aceptado: 02/08/016

El autor es Psicólogo del Deporte, Presidente de la Sociedad Latinoamericana y del Caribe de Psicología de la Actividad Física y del Deporte (SOLCPAD).

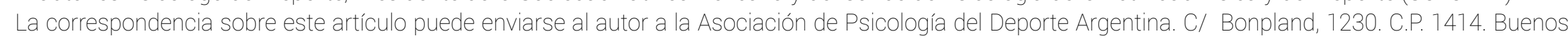
Aires, Argentina. E-mail: 
Tras más de 20 años de experiencia profesional como psicólogo del deporte, habiendo trabajado con equipos de fútbol de Argentina de primera división " $\mathrm{A}$ " y "B" (Ferrocarril Oeste, Chacarita Juniors, Independiente de Avellaneda, Vélez Sarsfield, Platense, UAI Urquiza y Atlanta), en México con el Club Toluca (primera división) y la Selección Nacional Juvenil (sub-17 y sub-20) y Absoluta de Argentina, mi experiencia me dice que no es lo mismo trabajar con adultos, juveniles, en un club o una selección. He tenido la posibilidad de intervenir en todas estas instancias y a nivel de la selección nacional, la presión es mucho mayor (Roffé y García-Ucha, 2005). Son muy pocos los futbolistas que rinden más o igual en la selección que en su club, principalmente asociado a un componente emocional (Roffé, 2004). Por ejemplo, por una cuestión cultural (países con una alta pasión por el fútbol o la repercusión de los éxitos y fracasos de la selección en el país), lo que puede representar jugar en la selección para el jugador (importancia, responsabilidad o identidad nacional) y las circunstancias de la propia competición (eliminatorias para la clasificación de una gran cita deportiva o jugar contra los mejores como exigencia deportiva), entre otras cuestiones.

Por ello, la preparación psicológica es fundamental para el alto rendimiento en fútbol, tal como indica la amplia bibliografía al respecto (García-Naveira y Jerez, 2012; Morilla et al., 2003; Rivas y Aragón, 2003; Roffé y Rivera, 2014; Vives y Garcés, 2003), en el que en una selección nacional cobra un especial interés dado las demandas específicas de la competición (partidos cada ciertos meses en formato de eliminatorias o partidos amistosos, y en Mundiales o Copa America cada 3 o 4 días) y los condicionantes propios de la concentración (viajes, estancias, etc.). Además, cabe destacar como el rol y labor del psicólogo del deporte se tiene que adaptar a estas circunstancias como veremos más adelante.

A pesar de ello, no existe mucha historia y tradición de que las selecciones nacionales de fútbol lleven un psicólogo especializado en deporte a la máxima cita como es un mundial. La investigación nada sencilla (debido al anonimato o confidencialidad profesional) a través de lo que se conoce del medio nos brinda unos datos (según nuestra investigación en internet, hablando con colegas y organizaciones de Psicología de todo el mundo): 9 de 32 selecciones nacionales contaron con personas encargadas de la parcela psicológica en el Mundial de Fútbol de Brasil 2014, en el que solo 3 de ellos eran psicólogos con formación y experiencia en alto rendimiento deportivo (ver Tabla 1).

Una vez contextualizado mi labor, lo primero que necesito escribir es que para poder implementar la intervención psicológica como complemento y parte de un trabajo interdisciplinar con el cuerpo técnico y servicios médicos, se tienen que dar varios factores. Me refiero a que exista un
Tabla 1. Profesionales que se han ocupado de la parcela psicológica en las Selecciones Nacionales en el Mundial de Fútbol de Brasil 2014

\begin{tabular}{ll}
\hline Profesión & País \\
\hline Psicólogo del Deporte & $\begin{array}{l}\text { Alemania (Hans-Dieter Hermann), } \\
\text { Brasil (Regina Brandao) y Colom- } \\
\text { bia (Marcelo Roffé) }\end{array}$ \\
\hline Psiquiatra & Argelia, Inglaterra e Italia \\
\hline Psicólogo Clínico & Nigeria y México \\
\hline Sociólogo & Costa Rica \\
\hline
\end{tabular}

líder que le brinde al psicólogo deportivo el lugar justo, desprovisto de urgencias y en el marco de un proyecto, valorando su saber-hacer en el área del entrenamiento mental (Olmedilla, García y Garcés, 1998; Olmedilla, Ortín, Andréu y Lozano, 2004)

Este trabajo psicológico no es para cualquier entrenador, cuerpo técnico o grupo de jugadores. Ni para cualquier dirigente. En este caso, partiendo del liderazgo y convencimiento del entrenador José Pekerman en los beneficios de la preparación psicológica y la inclusión del psicólogo del deporte a partir de buenas experiencias previas conmigo (campeonatos mundiales, panamericanos y sudamericanos con la selección Argentina juvenil sub 17 y sub 20, 5to puesto en el Mundial de Alemania del 2006 con la Selección Absoluta de Argentina, 2 clasificaciones en la liguilla con el Toluca de México, etc.), todo es más fácil. El lugar que él le da al rol es decisivo. Pero no alcanza. Además tiene que haber un equipo de trabajo técnico-medico-administrativo que no sea prejuicioso y que entienda el aporte. Cuando ambas cosas están, llega lo fundamental: que el mensaje llegue a los jugadores y ellos lo tomen y se abran. Eso también sucedió. Y de entrada mucho tuvo que ver, en esa apertura, el "gran capitán" de la Selección, Mario Alberto Yepes, con más de 100 partidos en la Selección y 800 partidos en su carrera. Más que un capitán, un verdadero caudillo. Me refiero al hecho de su apoyo al trabajo de "Entrenamiento Mental", siendo quien guiaba y conducía al equipo.

Ahí las piezas empiezan a ordenarse. Por eso mi agradecimiento a todos. Jugadores, cuerpo técnico y dirigentes. Y a la Federación Colombiana de Fútbol, representada en sus dirigentes: siempre demostraron interés por esta especialidad. Sencillamente, gracias.

Por ello, el objetivo del presente trabajo es ofrecer al lector el trabajo psicológico realizado durante 3 años con la Selección Nacional de Colombia (2012-2015) y siendo muy cuidadoso con el secreto profesional (como lo he sido siempre), poder brindar algunos datos de lo vivenciado. 


\section{Experiencia profesional}

\section{Previa al mundial: hay que clasificar}

La demanda. Me parece muy relevante entender cuándo y por qué se incorpora mi trabajo. José Pekerman es un líder silencioso, muy trabajador y anti polémico. $Y$ muy cauto y prudente. Eso se lo da la sabiduría. Cuando recibí el llamado en enero del 2012 era para que supiera que me tenía en cuenta, pero como el sabiamente me dijo, aún no conocía a los jugadores. Colombia juega su primer partido (con él como entrenador) contra México en febrero del 2012 y gana. Y luego en junio es visitante por las eliminatorias contra Perú y Ecuador. Dos partidos durísimos. Gana el primero y pierde el segundo. Y en septiembre venia Uruguay de local y Chile de visitante, primero y segundo en la tabla de clasificaciones.

La demanda por parte de José fue: "te vas a sumar ahora, haremos cambios en la táctica, incorporaremos jugadores nuevos y arrancaremos con lo tuyo. Tenemos que hacernos fuertes de local, hacer de Barranquilla una fortaleza. Y empezar con Uruguay". Los objetivos solicitados eran hacer grupo, fortalecer la mente de los jugadores y aportarles mentalidad ganadora ante estos dos partidos y el resto de la clasificación, ya que Colombia no se había clasificado en los 3 mundiales anteriores. Estos datos indicaban que históricamente algo pasaba con la Selección de Colombia.

Convivencia en Madrid. Pensar que a José una parte de la prensa del país lo criticó porque no hizo partidos amistosos oficiales. Era fecha FIFA José y su cuerpo técnico decidieron ir y aprovechar Madrid para que los jugadores viajen menos y convivan más. Muchos juegan en Europa. La gran mayoría.

Los objetivos principales para esta estancia eran potenciar las relaciones y la unión en el equipo, realizar una presentación o acercamiento del trabajo psicológico para la mejora del rendimiento, haciendo hincapié en establecer objetivos y la ambición del equipo.

Esa convivencia la aprovechamos al máximo. En todas las aéreas. Y en la que a mí respecta, evaluamos (entrevistas, test, etc.) e intervenimos a nivel psicológico (talleres, dinámicas grupales, implementación de videos, etc.). Por ejemplo, con la presencia del cuerpo técnico y su aporte, realizamos una actividad en el que describimos los 16 secretos para llegar a Brasil. Los fuimos viendo y analizando uno por uno, no podemos nombrarlos por secreto profesional, pero se cumplieron la mayoría. Fuimos estableciendo metas y desarrollando un plan de acción para conseguirlos. Nos fuimos convenciendo.

La recepción fue estupenda. El jugador colombiano es muy educado y respetuoso. De hecho en el Hotel de Buenos
Aires previo al partido contra Argentina por eliminatorias, el personal del Hotel lo felicitó a José. Decían que pasaron muchos equipos nacionales e internacionales, pero ninguno tan educado.

Estaban abiertos para empezar a entender qué era el entrenamiento mental y qué se les podía aportar desde esta área (Roffé y Rivera, 2014). Fue un gran inicio. En palabras del periodista Hernandez-Bonnet (2015): "Pekerman convivía con sus futbolistas en una especie de retiro espiritual en el que el psicólogo jugó un papel preponderante para que los jugadores entendieran que a su talento natural, había que agregarle el poder de la mente".

La competición. José es un gran líder. Y como todo gran líder sabe esperar su oportunidad, no se apura. Así fue que durante tres años no dirigió luego del Tigres de Monterrey (México). Esperaba algo que lo satisficiera. Ofertas le llovían. Pero a todas les decía que no. Alguien que le dijo que NO dos veces a la Selección Absoluta de Argentina, puede decirle que no a cualquier ofrecimiento.

Cuando llega esta oportunidad que él alguna vez había visualizado, además de lo afectivo porque vivió en Colombia (como jugador y finalizó su carrera deportiva allí por una lesión en la rodilla), y una de sus hijas nació en aquel país, se da cuenta que le motiva el desafío y Colombia tiene con que ir a buscar lo que tanto añoraba: clasificar, luego de 3 mundiales sin hacerlo. Había mucho por ganar. Era un reto para él.

Dijimos que Colombia podía hacerse fuerte de local. Que había nuevos jugadores y que empezábamos a cambiar la mentalidad. De a poco. Esto es trabajo, se requiere tiempo y un proyecto, no es magia.

Llega Uruguay: era una linda oportunidad para pisar fuerte, ante un gran rival. Más allá de la goleada de 4 a 0 , impensada para muchos, cabe destacar la determinación y decisión con que salió el equipo. La misma que José les inculcó. Gol de Falcao a los dos minutos. Ya era otro partido. Teo Gutiérrez, figura y autor de dos goles, apenas termina el partido agradece por televisión al cuerpo técnico y en especial a la ayuda psicológica que recibió y nombra la palabra "visualización".

Empezar a creer. Según los especialistas José le dio a este equipo tres cosas: orden táctico, trabajo en equipo y mentalidad ganadora. No es todo psicológico.

Todos decían y repetían: Colombia tiene enormes jugadores. Pero la pregunta era ¿pero entonces porque no llega al mundial? ¿Qué le falta? ¿Qué es lo que no funciona? ¿Porque siempre les falta uno o dos puntos para clasificarse? Como señala Kerr (2014) "una colección de individuos talentosos pero sin disciplina personal, en última instancia fracasará inevitablemente. La personalidad triunfa sobre el talento". 
Este sistema de eliminatorias que dura más de dos años tiene sus pros y sus contras. Entre las contras es que al ser tan largo es muy difícil mantener el mismo rendimiento durante dos años y medio seguidos. Además, cada jugador viene a la Selección con sus propias circunstancias de club (cansancio, titularidad o suplencia, rendimiento, etc.). Inevitablemente hay altibajos.

Luego de Uruguay, mantener la humildad era la clave. Chile esperaba de local y descansado, primero en la tabla y sin haber jugado antes por fecha libre del calendario de la FIFA.

Muchos imaginaban a una Colombia que iba a sacar un punto. José decidió otras cosas: vamos a por los tres puntos, a sorprenderlos. El equipo volvió a brillar y superando la adversidad (que no había podido remontar contra Ecuador antes de comenzar nuestro trabajo), tras ir perdiendo 1-0, dio vuelta el resultado y venció 1 a 3 . Seis puntos de seis. Algo estaba cambiando. Siguiendo a Olivares y Méndez (2010), comenzamos a trabajar en el Modelo de Murphy de Afrontamiento, y con las estrategias de: reducir la amenaza, distracción, evitación y contener la ira.

También, dentro del trabajo realizado, quiero destacar la labor en cuanto al establecimiento de unos valores que definiera el grupo y la cohesión, la evaluación psicológica y la gestión de las presiones.

Valores y cohesión grupal. La preparación psicológica de cada partido tenía una serie de acciones incorporadas, algunas de ellas con la presencia y participación del cuerpo técnico, en formato de presentación y/o vídeo. A modo de ilustración, se describe un breve esquema de una de ellas: a) presentación de 20 diapositivas en power point (con slides alusivas a un tema elegido junto a José, por ejemplo concentración bajo presión), b) un video de 7 minutos o dos de 3 ó 4 minutos (alusivos al tema para ejemplificarlo), c) la realización de un trabajo entrelazado en 4 grupos sobre la cuestión planteada (e.g., taller sobre el análisis de un texto que trataba sobre qué es ser un "ganador o perdedor"), d) las conclusiones finales en boca de José y a veces también del capitán, y e) posteriormente el quedarme a solas con los futbolistas para realizar un juego o dinámica grupal con el objetivo de que se conocieran más y el grupo se fuera cohesionando con mayores detalles, hasta ser un verdadero equipo. A su vez salía una información muy valiosa que, amparado en el secreto profesional, me permitía ayudarlos mejor grupal e individualmente. $Y$ también para descargar tensiones que las hay y muchas en alto rendimiento.

Un taller que quedó en la memoria e historia "viva "del equipo, fue el que desarrollamos bajo la consigna ¿por qué elegiste ser futbolista? Cada jugador fue contando uno por uno su elección, su infancia, su origen humilde (90 por ciento del equipo), y esto fue muy emotivo y motivador, cono- cer más sobre el compañero, para entenderlo más, quererlo más y unirse más, dando la vida por él dentro de la cancha.

\section{Evaluaciones realizadas}

Como comentaba en la introducción, el futbol profesional es diferente a fútbol juvenil, y la selección es diferente a los clubes. Esta distinción la he notado cuando se ha planteado realizar algún tipo de evaluación e intervención. Por ejemplo, en juveniles es más fácil realizar una evaluación psicológica debido a que son jóvenes, más dóciles, liberados y predispuestos. Con los profesionales resulta más complicado, tienen más reparo, son más herméticos, tal vez por falta de confianza e información (aspectos que tenemos que construir como profesionales), en el que además, al ser una selección, no hay un trabajo regular del "día a día", y si de concentración en concentración o de campeonato en campeonato. Es bueno tener clara estas diferencias. Desde la confianza, confidencialidad y credibilidad que fui ganando poco a poco con mi trabajo, se realizó una labor de concienciación y participación de la importancia de poder evaluar como punto de referencia para la mejora o el seguimiento. En ese sentido, se ha evaluado características de personalidad (e. g., rasgos de personalidad), habilidades psicológicas (e. g., relajación, visualización y concentración), motivaciones (e. g., establecimiento de objetivos), vínculos entre los jugadores (sociograma), el rendimiento deportivo (e. g., conductas deportivas), etc. (Díaz-Morales y García-Naveira, 2001; Roffé, 2009).

Intervenciones anti-estrés. Es importante trabajar la autoconfianza y el control del estrés (González-Campos, Valdivia-Moral, Zagalaz y Romero, 2015). Dada la importancia del manejo de las presiones, el aprendizaje de habilidades de afrontamiento para reducir el estrés se integró dentro de los talleres con los jugadores. Partiendo de mi experiencia previa con la selección sub-20 de Argentina que quedó campeona del mundo en el año 2001, en el que se trabajó en esta línea (Roffé, 2003), se introdujo las técnicas de respiración y visualización. Habitualmente se trabajaba el día antes de la competición. La visualización bien utilizada, es una técnica cognitiva que puede ser decisiva para los futbolistas, mejorando la concentración y la confianza, mientras que con la relajación lográbamos reducir la ansiedad y un mejor descanso/sueño (Roffé y García-Ucha, 2005).

También se entrenó la resiliencia mental (Ruiz-Barquín, De la Vega-Marcos, Poveda, Rosado y Serpa, 2012) ya que es una de las variables claves para hacer de un equipo, un equipo ganador. Por un lado, se desarrollaron talleres y actividades para afrontar situaciones adversas o de frustración, con el objetivo de afrontarlas, resolverlas, anticiparse 
de cara al futuro y fortalecerse mentalmente. Por otro, las propias situaciones deportivas y de la competición te van formando y preparando (e.g., afrontando marcadores en contra en los partidos). Para ejemplificar este punto, destacar las remontadas ante Chile (se remonta un 0-3 y se empata 3-3 y se clasifica al Mundial) y Paraguay (se remonta un 0-1 y se gana 2-1 de y se queda como cabeza de serie).

Además se trabajó la mentalidad ganadora. La mentalidad ganadora que con José transmitíamos incluía los siguientes ítems que construimos nosotros, entre cuerpo técnico y futbolistas a partir de un taller.

- Ser protagonistas

- No subestimar a nadie

- Nunca bajar los brazos

- Estar enfocados en la tarea

- No quedar enganchados al error

- Tomar decisiones, no dudar

- Ser solidarios con el compañero

- Tener metas claras de equipo sin perder las metas individuales

- Auto dialogo y pensamiento positivo

- Nunca conformarse

- Manejar las presiones

- Fluir

- Tener buena comunicación entre ellos y con cuerpo técnico y medico

- Ser solidario con el compañero

\section{Mundial 2014: Brasil es la cita}

Luego de 3 mundiales sin clasificar, Colombia vuelve al primer plano Mundial. La alegría de haber clasificado era enorme, pero ya era historia. Había que revalidar las estadísticas: Colombia llega al mundial entre los 5 mejores equipos del mundo según el ranking FIFA. Se podía ir a pasear y conformarse o ir con ambición a "dejar huella". El entrenador elige la segunda opción.

Primer obstáculo: lesiones y líderes ausentes. Radamel Falcao García fue, es y será un ídolo para Colombia. Su lesión le impidió llegar en buena forma a un campeonato tan exigente. La ilusión estaba. Sus palabras y como lo enfrentó fueron ejemplares.

El equipo lo quiere mucho. Fue muy duro sobrellevarlo. Lo mismo lo de Amaranto Perea, otro líder -referente. Muy querido en el equipo. Los dos hicieron mucho para que Colombia llegue al Mundial.

Y otro gran jugador y excelente persona que se lo pierde y a último momento por una lesión fue Aldo Leao Ramírez, duro para todos, ya que por edad (33 años) como Amaranto, "era" su primer y último Mundial.
Se trabajó y se asumió individual y grupalmente. Desde una perspectiva de aceptación y compromiso de la situación (Soriano y Valdivia, 2006) había que seguir, por ellos también. Era tan buena la química que existía que Falcao y Perea viajaron a ver el debut de la selección ante Grecia.

El mundial. A todos nos miden por resultados. Se batieron varios records propios:

- Ganar dos partidos seguidos (sobre un total de 4)

- Cantidad de goles a favor (12 en 5 partidos)

- Clasificar solo en dos partidos jugados (quedaba uno por jugar) a octavos de final.

- El goleador del torneo (James Rodriguez con 6 goles)

- El mejor gol del Mundial: James a Uruguay

- El premio Fair Play

- El record de Faryd Mondragon de jugar con 42 años un mundial.

- Los 100 partidos de Mario Yepes con la Selección Absoluta

- El $5^{\text {to }}$ puesto por primera vez en su historia.

Los resultados fueron:

COLOMBIA 3-GRECIA 0 (grupo)

COLOMBIA 2-COSTA DE MARFIL 1 (grupo)

COLOMBIA 4-JAPON 1 (grupo)

COLOMBIA 2-URUGUAY 0 (octavos de final)

COLOMBIA 1-BRASIL 2 (cuartos de final)

El trabajo psicológico. Concentrábamos en el predio del club San Pablo. Espectacular. Muy bien elegido realmente. Esto beneficiaba al equipo en la paz cotidiana del trabajo y en la facilidad para movilizarnos.

El trabajo psicológico durante el mundial se centró en dar respuesta a las demandas individuales (e.g., contener a los jugadores que estaban algo más intranquilos de lo habitual ante un partido) y fortalecer el grupo. Se continuaron realizando dinámicas grupales ya comentadas de mi intervención, y además concursos de baile, juegos de comunicación, dinámicas de cohesión, realización de carteles individuales motivacionales, etc. Estas actividades tenían como objetivo unir más al grupo a partir de un mayor interacción con el otro, una búsqueda de identidad y pertenencia. Así como descargar tensiones que las hay y muchas.

En lo personal sentí que me preparé durante 20 años de trabajo de campo, para aportar un "granito de arena" en ese momento. Me había quedado la frustración de no haber viajado al mundial de Alemania 2006 con la Selección Argentina (estuve como asesor externo), 8 años antes, y el fútbol me dio revancha con otra camiseta.

Lo disfruté. Era mi sueño. Lo que me faltaba. Y sentí que mi aporte fue valorado. Fueron 2 años de trabajo previos y 45 días concentrados con ellos en el Mundial. Como uno más. Me refiero a tres años de trabajo ya que renuncié a la 
Selección luego de la Copa America 2015 en junio del 2015, decisión que me costó mucho tomar. Luego de 15 años maravillosos de trabajo con Pekerman donde nos enriquecimos mutuamente y aprendí mucho, sentí que había finalizado un ciclo y que necesitaba nuevos desafíos. Y seguramente haber trabajado en un MUNDIAL que era mi sueño, ya estaba cumplido.

Relación con la prensa. Es bueno destacar que en el camino del psicólogo deportivo en el alto rendimiento, el mismo sufre muchas presiones y a su vez muchas tentaciones. Entre las tentaciones está la prensa. Por eso consideramos que el profesional debe tener conocimiento, experiencia, formación constante, supervisión con un colega calificado, posición ética, neutralidad y análisis personal. Con todos estos elementos reduce su margen de error, aunque en tanto humano, será imposible que no se equivoque.

Respecto de este trabajo puntual que estamos describiendo, en estos tres años rechacé infinitas notas radiales, televisivas y escritas de los mejores medios colombianos e internacionales. No brindé una nota siquiera. La decisión fue tomada desde el compromiso con el trabajo y con la eficacia de la intervención, aunque consideramos que el cuerpo técnico si tiene que valorar, reconocer y nombrar a sus todos sus ayudantes "invisibles" en algún momento, ya que no estoy de acuerdo en la postura de "esconder al psicólogo deportivo", ya que resulta imposible lograrlo y además persigue un fin muy mezquino. Este último elemento nos parece importante dejarlo planteado para la discusión y poder retomarlo.

\section{Discusión}

Según mi experiencia profesional, la edad ideal para la intervención psicológica en cuanto a desarrollo y preparación mental del jugador de fútbol es entre los 13 y 21 años, aunque con deportistas adultos de alto rendimiento se puede realizar un muy buen trabajo en materia de entrenamiento mental para optimizar los recursos psicológicos y afrontar la competición. La suerte del psicólogo del deporte es trabajar con deportistas talentosos, que sean buenas personas, educados, predispuestos y respetuosos. Todo esto facilita. Y esto sucedió.

Mis colegas de la Asociación de Psicología del Deporte Argentina (APDA) me hicieron ver que fui el primer psicólogo del deporte argentino en formar parte de una selección en un mundial de mayores. Ojala sean muchos en el futuro. Es una experiencia única, maravillosa e inolvidable. Quizás homologada por los Juegos Olímpicos.

En equipo se disfruta más, somos un complemento del entrenador y del cuerpo médico, el psicólogo deportivo solo no puede hacer nada. Como dice un proverbio africano, "si quieres ir rápido ve solo, si quieres ir lejos ve acompañado". El trabajo en equipo e interdisciplinar es una cuestión básica para el éxito colectivo.

Y haber sido parte de ese equipo que dejó una huella imborrable, nunca lo olvidaré. Gracias a los jugadores (me costó mucho dejarlos), al gran capitán Yepes, y al cuerpo médico y técnico y administrativo. Y a la gente de Colombia, que a nuestro regreso a BOGOTA nos hizo sentir como si hubiéramos salido "campeones del mundo". Éramos 23 integrantes del cuerpo técnico-medico-administrativo para 23 jugadores. Nada es casual, (había hasta cocinero propio, fisiólogo, editor de videos, tres ayudantes tácticos, etc.).

A favor es que hacía 14 años que venía trabajando con Jose Pekerman y ese grado de confianza no tiene precio.

La fortaleza del trabajo podemos encontrarla en que se naturalizó la preparación mental a nivel de élite del futbol mundial, con gran recepción e inmejorables resultados. Como todos sabemos el preparador físico, el kinesiólogo o el médico no pueden no estar, pero el psicólogo deportivo sí. Ser uno más es la clave mas allá de los resultados, valorar los procesos, si los resultados acompañan, mucho mejor.

Como reflexiones finales quiero hacer referencia a algunos puntos que me resultan significativos: En primer lugar, no es lo mismo ser incorporado por el entrenador (eres parte del cuerpo técnico, con tus roles y funciones. Entra y sale con el técnico), por la federación (algo externo, con el gran reto de integrarse. Se puede ir el técnico y uno seguir) o por los servicios médico (perspectiva más clínica y no tanto de rendimiento). Aunque cada perspectiva te da y te quita, para el trabajo con una selección, el estar dentro del cuerpo técnico te consolida y beneficia profesionalmente.

Además, no es lo mismo venir trabajando con la selección hace 10 años como el colega alemán, que ser incorporado para el mundial. Todo proyecto requiere una planificación y tiempo de trabajo. Por ejemplo, Brasil no tuvo un psicólogo/a durante el Mundial. Regina Brandao no estaba concentrada a tiempo completo los 45 días como el caso del psicólogo alemán o el mío. Ella era la psicóloga de Felipe Scolari (seleccionador de Brasil). Intervino con los jugadores antes del partido de Colombia y antes de Alemania (con quien Brasil sufre la peor derrota en un mundial) por pedido del director técnico. Y de alguna manera, Regina quedó muy expuesta por la prensa, siendo lo que es: una gran profesional. Detalle para retomar en otra discusión que se enlaza con el planteado anteriormente: ni exponerlo ni esconderlo.

También podemos preguntarnos porque potencias como Holanda, Argentina, España y equipos importantes como Uruguay y Chile no cuentan en la selección absoluta con el aporte del psicólogo, aunque si en categorías juveniles. Son 
espacios que tenemos que seguir conquistando desde la difusión de nuestra labor y el trabajo bien hecho. Y sobre todo, llegar a los entrenadores.

Dentro de mi labor profesional, como aprendizaje destacaría la importancia de la alianza estratégica con el/ los capitán/ es para gestionar el grupo y ser mediador para fortalecer aún más el vínculo entrenador-capitán/ es. Esta función facilita la inserción del trabajo psicológico del psicólogo y el buen desempeño del equipo.

Por otro lado, me gustaría destacar el perfil de personalidad del jugador colombiano (educados y con apertura a la mejora) que le posibilita prepararse para conseguir nuevos retos y objetivos. Por ello, hay que formar personas y deportistas desde edades tempranas, como un todo e indivisible (García-Naveira y Jerez, 2012).

Además, la adaptación de la labor del psicólogo en cuanto a tiempos y contextos de trabajo. Por un lado, hay unos calendarios establecidos por la FIFA, en el que cada cierto mes las selecciones tienen partidos amistosos o de competición (e. g., clasificación para el mundial). También está el gran evento en sí (e. g., el Mundial). Yo viajaba y concentraba con el equipo ( 7 a 11 días en el primer caso y 45 días en el segundo), buscaba salas para intervenir con el grupo y de forma individual, me relacionaba con el resto de profesionales, hacíamos equipo. Por otro, realizaba un seguimiento de los jugadores vía online (e.g., por skype) durante los períodos entre concentraciones (ya que podrían pasar unos 2-3 meses hasta que nos volviésemos a ver).

Para terminar, decir que las perspectivas futuras en el trabajo con selecciones nacionales deberían ser cada vez mejores si seguimos haciendo bien nuestro trabajo, asesorando con formación, ética, perfil bajo, esfuerzo, trabajo en equipo, ubicuidad, pasión y humildad. Y sobre todo, comprender que ser psicólogo del deporte es una profesión especializada, con todo lo que ello conlleva (Cantón, 2010).

Las limitaciones podemos encontrarlas en que como son actividades "pioneras" a nivel elite no existe una base de datos o fuentes o profesionales con quienes investigar, comparar, crecer y revisar aciertos y errores.

\section{Referencias}

Cantón, E. (2010). La Psicología del Deporte como profesión especializada. Papeles del Psicólogo, 37, 237-245.

Díaz Morales, J. F. y García Naveira, A. (2001). Evaluación de metas en jugadores de fútbol de categoría juvenil y absoluta. Revista de psicología del deporte, 10, 211-224.
García-Naveira, A. y Jerez, P. (2012). Departamento de Psicología del Club Atlético de Madrid: filosofía, programación y desempeño profesional en el fútbol base. Cuadernos de Psicología del Deporte, 12(1), 111-120.

González-Campos, G., Valdivia-Moral, P., Zagalaz, M. L. y Romero, S. (2015). La autoconfianza y el control del estrés en futbolistas: revisión de estudios. Revista Iberoamericana de Psicología del Ejercicio y el Deporte, 10, 95-101.

Hernandez-Bonnet, J. (2015). El Método Pekerman. Madrid, España: Edición Planeta.

Kerr, J. (2014). El legado, 15 lecciones sobre liderazgo, que nos enseñan los ALL BLACKS sobre la empresa de vivir. Buenos Aires, Argentina: Editorial Club House.

Morilla, M., Pérez, E., Gamito, J., Gómez, M., Sánchez, J. y Valiente, M. (2003). Planificación psicológica de la cantera del Sevilla F.C. S.A.D.: organización, funcionamiento y programa deportivo-formativo. Cuadernos de Psicología del Deporte, 3 (2), 17-30.

Olivares, J. y Méndez, F. (2010). Técnicas de modificación de la conducta. Madrid, España: Biblioteca Nueva.

Olmedilla, A., García, C. y Garcés, E. (1998). Un análisis del papel profesional del psicólogo del deporte desde la percepción del entrenador de fútbol. Revista de Psicología del Deporte, 7, 95113.

Olmedilla, A., Ortín, F., Andréu, M. y Lozano, F. (2004). Formación en psicología para entrenadores de fútbol: una propuesta metodológica. Revista de Psicología del Deporte, 13, 247-262.

Rivas, M. y Aragón, M. (2003). Intervención Psicológica en el fútbol base: un trabajo en el club América de México. Cuadernos de Psicología del Deporte, 3(2), 35-40.

Roffé, M. (2003). La preparación psicológica de la Selección Juvenil Argentina Sub-20 de fútbol para el Mundial 2001: un año de trabajo con futbolistas de elite. Efdeportes, 67. Recuperado de http://www.efdeportes.com/efd67/psfutbol.htm

Roffé, M. (2004). Como evaluar las presiones y miedos de los deportistas de Selección: una experiencia de 4 años con futbolistas juveniles. Efdeportes, 72. Recuperado de http://www. efdeportes.com/efd72/presion.htm

Roffé, M. (2009). Evaluación psicodeportologica: los 30 test psicométricos y proyectivos. Buenos Aires, Argentina: Lugar Editorial.

Roffé, M. y García-Ucha, F. (2005). Alto rendimiento, Psicologia y deporte. Buenos Aires, Argentina: Lugar editorial.

Roffé, M. y Rivera, S. (2014). Entrenamiento mental en el futbol Moderno. Madrid, España: Futbol de Libro.

Ruiz-Barquín, R., De la Vega-Marcos, R., Poveda, J., Rosado, A. y Serpa, S. (2012). Análisis psicométrico de la escala de resiliencia en el deporte del fútbol. Revista de Psicología del Deporte, 21, 143-151.

Soriano, M.C. y Valdivia, M. (2006). La terapia de aceptación y compromiso (ACT). Fundamentos, características y evidencia. Papeles del Psicólogo, 27, 79-91.

Vives, L. y Garces, E. (2003). Intervención psicológica en un club de fútbol base: propuesta de un sistema de actuación psicológica desde sus diversas áreas. Cuadernos de Psicología del Deporte, 3(2), 51-66. 